\title{
Use of pyrosequencing and DNA barcodes to monitor variations in Firmicutes and Bacteroidetes communities in the gut microbiota of obese humans
}

\author{
Fabrice Armougom and Didier Raoult*
}

\author{
Address: URMITE - UMR CNRS 6236, IRD 3R198, Université de la Méditerranée, Faculté de médecine, 27 Boulevard Jean Moulin, 13005 Marseille, \\ France \\ Email: Fabrice Armougom - fabrice.armougom@univmed.fr; Didier Raoult* - didier.raoult@gmail.com \\ * Corresponding author
}

Published: I December 2008

BMC Genomics 2008, 9:576 doi:10.1186/147|-2164-9-576
Received: 18 June 2008

Accepted: I December 2008

This article is available from: http://www.biomedcentral.com/147I-2/64/9/576

(c) 2008 Armougom and Raoult; licensee BioMed Central Ltd.

This is an Open Access article distributed under the terms of the Creative Commons Attribution License (http://creativecommons.org/licenses/by/2.0), which permits unrestricted use, distribution, and reproduction in any medium, provided the original work is properly cited.

\begin{abstract}
Background: Recent studies of $16 \mathrm{~S}$ rRNA genes in the mammalian gut microbiota distinguished a higher Firmicutes/Bacteroidetes ratio in obese individuals compared to lean individuals. This ratio was estimated using a clonal Sanger sequencing approach which is time-consuming and requires laborious data analysis. In contrast, new high-throughput pyrosequencing technology offers an inexpensive alternative to clonal Sanger sequencing and would significantly advance our understanding of obesity via the development of a clinical diagnostic method. Here we present a cost-effective method that combines I6S rRNA pyrosequencing and DNA barcodes of the Firmicutes and Bacteroidetes $16 \mathrm{~S}$ rRNA genes to determine the Firmicutes/Bacteroidetes ratio in the gut microbiota of obese humans.
\end{abstract}

Results: The main result was the identification of DNA barcodes targeting the Firmicutes and Bacteroidetes phyla. These barcodes were validated using previously published I6S rRNA gut microbiota clone libraries. In addition, an accurate F/B ratio was found when the DNA barcodes were applied to short pyrosequencing reads of published gut metagenomes. Finally, the barcodes were utilized to define the F/B ratio of $16 \mathrm{~S}$ rRNA pyrosequencing data generated from brain abscess pus and cystic fibrosis sputum.

Conclusion: Using DNA barcodes of Bacteroidetes and Firmicutes 16 S rRNA genes combined with pyrosequencing is a cost-effective method for monitoring relevant changes in the relative abundance of Firmicutes and Bacteroidetes bacterial communities in microbial ecosystems.

\section{Background}

Investigations of the bacterial $16 \mathrm{~S}$ rRNA genes play an essential role in the exploration of microbial diversity and bacterial taxonomy. The composition of bacterial communities is typically studied by implementing clonal Sanger sequencing of $16 \mathrm{~S}$ rRNA PCR products [1-4]. In humans, the ratio of Firmicutes to Bacteroidetes (F/B ratio) was found to be significantly higher in obese individuals than in lean individuals. Ley and colleagues [4] demonstrated that a decrease in the $\mathrm{F} / \mathrm{B}$ ratio in obese individuals correlated with weight loss over time. The authors suggested that modulation of the abundance of particular bacterial communities inherent to the gut microbiota would be beneficial for the treatment of obesity. In these 
experiments, the composition of the gut microbiota was monitored over a one-year period using a culture-independent method based on shotgun sequencing of $16 \mathrm{~S}$ rRNA clone libraries. Although this method was successfully used for taxonomy characterization at the species level, it was a time-consuming and expensive [5] process that required the application of exceedingly laborious data analyses. The development of an inexpensive and quick clinical diagnostic method would significantly improve our understanding of obesity, which is a common health issue affecting a large numbers of individuals.

In this regard, the new high-throughput technology of pyrosequencing [6] offers a cost-effective alternative to traditional sequencing methods, particularly for metagenomic studies, but also for $16 \mathrm{~S}$ rRNA-based microbial diversity studies [7-9]. A comparison of the cost and highthroughput capacity of sequencing technologies indicated that 454-Roche pyrosequencing generated far more sequence data per run at a much lower cost (30 times) than conventional dye-terminator sequencing [10]. The short length of reads (100-250 base pairs) generated by this new high-throughput technology, however, limits full length bacterial 16S rRNA sequence assembly; thus, bacterial taxonomy characterization of mixed microbial samples remains a daunting task with the risk of chimera production. In order to address this problem, recent studies have reported efficient methods for classifying short sequences [11-13] at the phylum or genus taxonomic level. In addition, short DNA-specific regions that exhibit significant variability between bacterial species have been recently investigated to avoid the computational challenge of full-length 16S rRNA sequence assembly $[5,8,14,15]$. Thus far, however, the sensitivity of the primers used to target short specific or variable regions over the entire bacterial domain is unclear due to the lack of exhaustive $16 \mathrm{~S}$ rRNA sequence testing.

Since Firmicutes and Bacteroidetes are the main bacterial phyla involved in alterations of the gut microbiota in obese individuals, it would be useful to develop a faster and less expensive method for monitoring variations in their relative abundance. In this study, we report a method for rapidly estimating the ratio of Firmicutes to Bacteroidetes using thousands of pyrosequencing reads generated from near-full 16S rRNA gene amplification products or complete bacterial metagenomes. We identified two DNA barcodes as genomic signatures specific to the 16S rRNA genes of species belonging to the Bacteroidetes or Firmicutes phylum. Such DNA barcodes for bacterial phyla are not short sequence tags added to PCR products of microbial samples for massive parallel or barcoding pyrosequencing investigations $[5,15]$.
One of the major benefits of these DNA barcodes was the ability to rapidly provide an accurate $\mathrm{F} / \mathrm{B}$ ratio from a pool of thousands of short sequencing reads generated by the pyrosequencing method, without the need to assemble sequences, perform multiple sequence alignments, generate phylogenetic reconstructions, or perform BLAST analyses. Therefore, our DNA barcodes used in combination with pyrosequencing technology will be useful for clinical diagnosis and for studies involving a large spectrum of subjects and conditions such as exhaustive caloric intake and antibiotic effects.

\section{Results \\ DNA barcodes}

The Bacteroidetes DNA barcode is a specific sequence of 12 nucleotides, while the Firmicutes barcode is a degenerate sequence composed of 26 nucleotides (Table 1 ). Based on the 16S rRNA gene sequence from $B$. fragilis (RDP-II accession number: S000000037), the Bacteroidetes DNA barcode spans base pairs 537 to 548. The Firmicutes DNA barcode spans base pairs 1,163 to 1,188 of the $16 \mathrm{~S}$ rRNA gene from C. difficile (RDP-II accession number: S000260455).

\section{Sensitivity and specificity of the DNA barcodes}

Figure 1 shows the high sensitivity inherent to the use of DNA barcodes from Bacteroidetes and Firmicutes phyla when applied to the Ribosomal Database Project [16] (RDP-II) and the Greengenes [17] database. The Bacteroidetes DNA barcode matches $96.52 \%$ and $93.60 \%$ of the $16 \mathrm{~S}$ rRNA sequences from the Bacteroidetes phylum in the RDP-II and Greengenes databases, respectively. Similarly, the Firmicutes DNA barcode matches $96.43 \%$ and $95.28 \%$ of the 16S rRNA sequences from the Firmicutes phylum in the RDP-II and Greengenes databases, respectively.

The Bacteroidetes DNA barcode is also highly specific given that $99.98 \%$ of the $113,00016 \mathrm{~S}$ rRNA sequences from the RDP-II database do not possess this DNA barcode (Table 2 ). There are actually only 18 Firmicutes sequences, two Planctomycetes sequences, and one Cyanobacteria sequence that possess the Bacteroidetes DNA barcode. Using the Greengenes database, the Bacteroidetes DNA barcode was less specific, demonstrating sequence matches with a greater number of phyla (7 of 15). The Firmicutes DNA barcode was also highly specific, with $98.25 \%$ of all tested $16 \mathrm{~S}$ rRNA sequences from the RDP-II database lacking

Table I: Sequence and length of the identified DNA barcodes

\begin{tabular}{ccc}
\hline Phylum & DNA barcodes & Length (bp) \\
\hline Bacteroidetes & GGGTTTAAAGGG & 12 \\
\hline Firmicutes & TCATGCCN[16]ACA & 26 \\
\hline
\end{tabular}




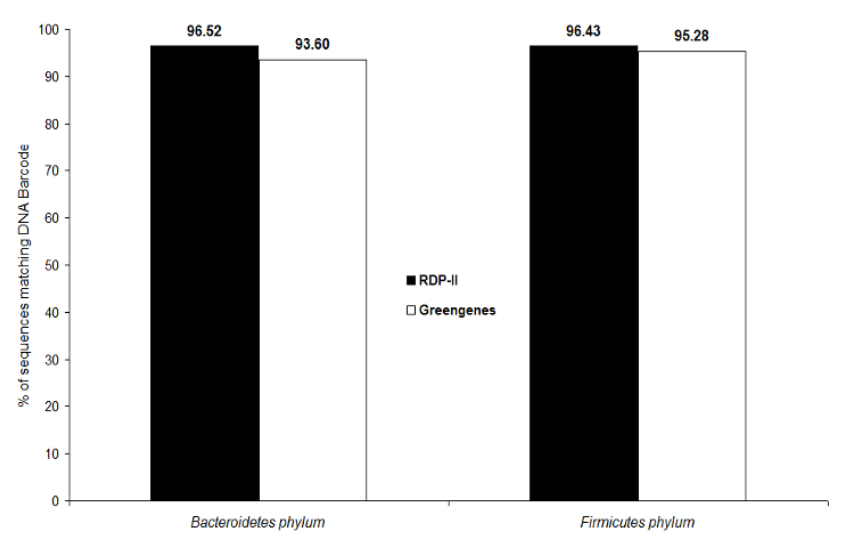

Figure I

Sensitivity of Firmicutes and Bacteroidetes DNA barcodes. RDP-II and Greengenes are I6S rRNA databases.

this DNA barcode. Compared to the Bacteroidetes DNA barcode, the loss in specificity observed with the Firmicutes DNA barcode is due to the fact that the latter matches $97.38 \%$ and $32.76 \%$ of sequences belonging to the Fusobacteria and Cyanobacteria phyla, respectively, using the RDP-II database (Table 2). However, These two phyla are poorly represented in the intestinal microbial flora (< $0.15 \%$ of the total $16 \mathrm{~S}$ rRNA sequences in each study) [14]. Finally, using the 335,830 sequences available in the RDP-II database, the false-discovery rates for the Firmicutes and the Bacteroidetes DNA barcode were found to be very low, $0.90 \%$ (3035 of 335830) and $0.06 \%$ (105 of 335830), respectively.

\section{Validation of DNA barcodes using full I6S rRNA gene sequences}

The 16S rRNA surveys based on the shotgun sequencing method from 16S rRNA clone libraries allow full 16S rRNA sequence assembly and can determine the relative abundance of a bacterial phylum using the phylogeny reconstruction ARB tool [18]. No significant statistical difference $(\mathrm{P}>0.05)$ was found when the DNA barcode method was used to assess Bacteroidetes and Firmicutes communities using four published 16S rRNA surveys of the gut microbiota (Table 3 ).

The first dataset $(11,831$ sequences) was obtained from a study of the diversity of human intestinal microbial flora [1] that demonstrated the Firmicutes and Bacteroidetes phyla represent $50.78 \%$ and $47.67 \%$, respectively, of all $16 \mathrm{~S}$ rRNA sequences. Applying our DNA barcode method to the same dataset, we observed that $50.36 \%$ and $47.52 \%$ of this dataset were represented by the Firmicutes and the Bacteroidetes phyla, respectively (Table 3 ). Thus, the percentages obtained for the two bacterial phyla using the
DNA barcodes or ARB tool were very close. A second dataset was obtained from a study of the gut microbiota of obese mice [3] and included 5,088 16S rRNA sequences. In this study, Ley et al. reported a 50\% reduction in the abundance of Bacteroidetes within obese mice compared to a control group of lean mice. For the subset of mice with the obese genotype, our estimate of the relative abundance of Bacteroidetes was $22.27 \%$, while the authors reported a relative abundance of $22.86 \%$. For the subset of mice in the control group (lean mice), our method provided a Bacteroidetes abundance estimate of $36.45 \%$, while the authors found a relative abundance of $36.39 \%$ (Table 3).

In another study, Turnbaugh and colleagues analyzed the increased capacity of the microbiome of obese mice to harvest energy from their diet [2]. From a dataset consisting of 4,157 $16 \mathrm{~S}$ rRNA sequences, the authors demonstrated that the relative abundance of Bacteroidetes in the obese donor and obese recipient groups was $28.74 \%$ and $31.88 \%$, respectively. For these two groups, our results indicated a nearly identical abundance of Bacteroidetes (29.04\% and 31.27\%, respectively). In addition, the relative abundance of Bacteroidetes in the lean groups was equivalent using these two methods. The relative abundance of Firmicutes for the two obese groups, however, was slightly different using our method (62.78\% and $60.32 \%$, respectively) and that reported by Turnbaugh et al. (67.86\% and 62.83\%, respectively) (Table 3 ).

The final dataset was retrieved from a survey of 16S rRNA sequences found in the gut microbiota of obese humans subjected to a specific calorie-diet [4] and monitored for the relative abundance of Bacteroidetes and Firmicutes. While the differences between our results and those of this study did not exceed $0.54 \%$ with regard to estimation of Bacteroidetes abundance, the relative abundance of Firmicutes observed using these two methods differed by up to 4.64\% (Table 3).

\section{Discrepancies}

There were only a few $16 \mathrm{~S}$ rRNA sequences that were resistant to equivalent taxonomic classification using the three analytical methods: the ARB tool, the DNA barcode method, and the RDP-II classifier. The vast majority of 16S rRNA sequences assigned to either the Firmicutes or Bacteroidetes phyla were similarly classified using the ARB tool (M1) or our DNA barcode (M2), therefore representing the "core" 16S rRNA sequence assignments (Figure 2, M1 n M2). In addition to this core, assignment of a small number of $16 \mathrm{~S}$ rRNA sequences to the Firmicutes and Bacteroidetes phyla was performed exclusively by one method or the other (Figure $2 \mathrm{M} 1 \backslash \mathrm{M} 2$ and $\mathrm{M} 2 \backslash \mathrm{M} 1$ ), and thus accounting for the discrepancies or ambiguous taxonomic assignments. 
Table 2: Specificity of the Firmicutes and Bacteroidetes DNA barcodes

\begin{tabular}{|c|c|c|c|c|c|c|}
\hline \multirow[b]{2}{*}{ Phylum } & \multirow[b]{2}{*}{$\begin{array}{l}\text { Number of } \\
\text { sequences in } \\
\text { RDP-II }\end{array}$} & \multirow[b]{2}{*}{$\begin{array}{l}\text { Number of } \\
\text { sequences in } \\
\text { Greengenes }\end{array}$} & \multicolumn{2}{|c|}{$\begin{array}{l}\% \text { of Hit with Bacteroidetes bar- } \\
\text { code }\end{array}$} & \multicolumn{2}{|c|}{$\%$ of Hit with Firmicutes barcode } \\
\hline & & & RDP-II & Greengenes & RDP-II & Greengenes \\
\hline Bacteroidetes & 18272 & 4718 & - & - & 0.41 & 0 \\
\hline Firmicutes & 45757 & 16023 & 0.04 & 0.057 & - & - \\
\hline Proteobacteria & 41277 & 38449 & 0 & 0.049 & 0.18 & 0.27 \\
\hline Actinobacteria & 11366 & 12736 & 0 & 0.0079 & 1.28 & 1.07 \\
\hline Cyanobacteria & 2164 & 1873 & 0.05 & 0.053 & 32.76 & 29.31 \\
\hline Spirochaetes & 1547 & 1677 & 0 & 0.059 & 0 & 0.18 \\
\hline Verrucomicrobia & 953 & 324 & 0 & 0 & 0 & 0 \\
\hline Planctomycetes & 864 & 553 & 0.23 & 0.54 & 0 & 0.18 \\
\hline Chloroflexi & 713 & 643 & 0 & 0 & 0 & 0.77 \\
\hline Acidobacteria & 782 & 865 & 0 & 0 & 0 & 0 \\
\hline Aquificae & 739 & 1131 & 0 & 0 & 0 & 0 \\
\hline Fusobacteria & 421 & 198 & 0 & 0 & 97.38 & 90.40 \\
\hline Nitrospirae & 376 & 156 & 0 & 0 & 0 & 0 \\
\hline Deinococcus-Thermus & 381 & 419 & 0 & 0.23 & 0 & 0.24 \\
\hline Chlamydiae & 178 & 167 & 0 & 0 & 0 & 0 \\
\hline Deferribacteres & 71 & 64 & 0 & 0 & 0 & 0 \\
\hline
\end{tabular}

RDP-II: Ribosomal Database Project II. For each bacterial phylum of the RDP-II and Greengenes databases, the percentage of sequences possessing the Bacteroidetes and/or the Firmicutes DNA barcode was calculated.

With regard to the $16 \mathrm{~S}$ rRNA data presented by Eckburg et al. [1], 17 of these sequences were assigned to the (Bacteroidetes) $\mathrm{M} 2 \backslash \mathrm{M} 1$ group, 62 were assigned to the (Firmicutes) $\mathrm{M} 2 \backslash \mathrm{M} 1$ group, and 12 were assigned to the (Firmicutes) $\mathrm{M} 1 \backslash \mathrm{M} 2$ group (Figure 2). The most significant discrepancy between M1 and M2 was observed with the $16 \mathrm{~S}$ rRNA data from Turnbaugh et al. [2]. In this report, 178 sequences were assigned to the (Firmicutes) $\mathrm{M} 2 \backslash \mathrm{M} 1$ group, 88 were assigned to the (Firmicutes) $\mathrm{M} 1 \backslash \mathrm{M} 2$ group, 14 were assigned to the (Bacteroidetes) $\mathrm{M} 1 \backslash \mathrm{M} 2$ group, and 41 were assigned to the (Bacteroidetes) M2 $\backslash \mathrm{M} 1$ group (Figure 3).
The "RDP-II Classifier" (RC) method was used to obtain additional 16S rRNA taxonomic assignments for the sequences defined as discrepancies between M1 and M2. We evaluated the agreement between the RPD-II classifier and the sequence assignments of $\mathrm{M} 1$ and $\mathrm{M} 2$.

The 12 sequences in the (Firmicutes) $\mathrm{M} 1 \backslash \mathrm{M} 2$ group (Figure 2 ), which were assigned to the Firmicutes phylum by our DNA barcode method, were classified into the Fusobacteria phylum (nine sequences) and into a group related to the Cyanobacteria phylum (three sequences) in the Eckburg et al. study. Similarly, analyses using the RC method resulted in the same classification as Eckburg's analysis with a bootstrap confidence (Bc) superior to $95 \%$, suggesting 
Table 3: Assessment of communities' abundance using clonal sequencing data

\begin{tabular}{|c|c|c|c|}
\hline Origin of data & Bacterial phylum & $\begin{array}{c}\text { Bacterial proportion (\%) found in } \\
\text { the study }\end{array}$ & $\begin{array}{c}\text { Bacterial proportion (\%) using } \\
\text { DNA barcode }\end{array}$ \\
\hline \multirow[t]{2}{*}{ Eckburg et al. study [I] } & Firmicutes & 50.78 & 50.36 \\
\hline & Bacteroidetes & 47.67 & 47.52 \\
\hline \multirow[t]{2}{*}{ Ley et al. mouse gut study [3] } & Bacteroidetes (+/+ Lean) & 36.39 & 36.45 \\
\hline & Bacteroidetes (Ob/ob Obese) & 22.86 & 22.27 \\
\hline \multirow[t]{8}{*}{ Turnbaugh et al. study [2] } & Firmicutes (Ob/ob Donors) & 67.86 & 62.78 \\
\hline & Bacteroidetes (Ob/ob Donors) & 28.74 & 29.04 \\
\hline & Firmicutes (Ob/ob recipients) & 62.83 & 60.32 \\
\hline & Bacteroidetes (Ob/ob recipients) & 31.88 & 31.27 \\
\hline & Firmicutes (Lean donors) & 54.82 & 54.82 \\
\hline & Bacteroidetes (Lean donors) & 40.96 & 40.96 \\
\hline & Firmicutes (Lean recipients) & 49.39 & 48.26 \\
\hline & Bacteroidetes (Lean recipients) & 47.7 & 46.69 \\
\hline \multirow[t]{8}{*}{ Ley et al. human gut study [4] } & Firmicutes ( 0 week on diet) & 88.47 & 84.97 \\
\hline & Bacteroidetes ( 0 week on diet) & 3.15 & 3.11 \\
\hline & Firmicutes (12 week on) & 85.35 & 82.16 \\
\hline & Bacteroidetes ( 12 week on diet) & 9.58 & 9.36 \\
\hline & Firmicutes ( 26 week on diet) & 70.91 & 66.97 \\
\hline & Bacteroidetes ( 26 week on diet) & 12.92 & 12.8 \\
\hline & Firmicutes (52 week on diet) & 75.3 & 70.66 \\
\hline & Bacteroidetes ( 52 week on diet) & 15.66 & 15.02 \\
\hline
\end{tabular}

Bacterial relative abundances found in four I6S rRNA surveys were compared to those inferred with the DNA barcodes application.

that our Firmicutes DNA barcodes provided 12 false-positive results. This result was not surprising given that our Firmicutes barcode matched a significant proportion of sequences belonging to the Fusobacteria and Cyanobacteria phyla in the RDP-II and Greengenes databases (Table 2). Moreover, the 62 sequences assigned to the (Firmicutes) $\mathrm{M} 2 \backslash \mathrm{M} 1$ group and the 17 sequences assigned to the (Bacteroidetes) $\mathrm{M} 2 \backslash \mathrm{M} 1$ group were similarly classified using the RC method, confirming the classification reported by Eckburg et al. and demonstrating that our
DNA barcode method failed only in the appropriate taxonomic assignment of a small number of sequences.

Based on the16S rRNA data obtained by Turnbaugh $e t$ al., seven sequences among the 41 sequences assigned to the (Bacteroidetes) M2 $\backslash \mathrm{M} 1$ group were not assigned by RC to the Bacteroidetes phylum as expected, but rather to the Firmicutes phylum ( $\mathrm{BC}>90 \%)$. Interestingly, these seven sequences also possessed the Firmicutes DNA barcode. In addition, of the 14 sequences assigned to the (Bacteroidetes) $\mathrm{M} 1 \backslash \mathrm{M} 2$ group, six sequences were also classified as 
DNA barcode (M1) Eckburg et al. data analysis (M2)

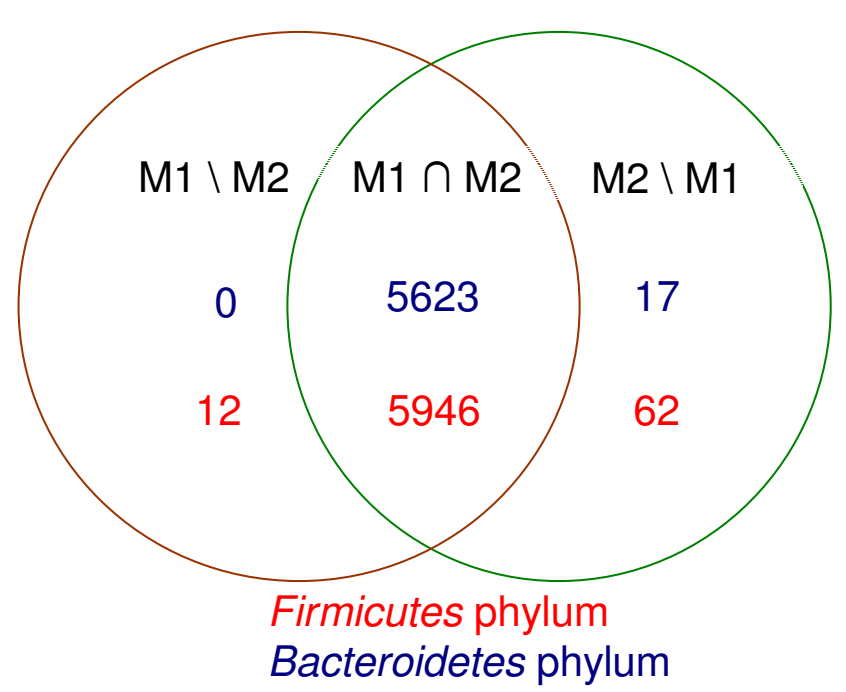

Figure 2

Discrepancies in sequence repartition of bacterial communities. $\mathrm{MI} \cap \mathrm{M} 2$ : in $\mathrm{MI}$ and $\mathrm{M} 2$; $\mathrm{MIIM2}$ : in $\mathrm{MI}$ out of M2; M2lMI: in M2 out of MI. The blue and red figures are the number of sequences, respectively classified in Bacteroidetes, Firmicutes phyla by MI or M2, or by both of them.

Bacteroidetes using the RC method ( $\mathrm{Bc}>90 \%$ ). Likewise, from the 178 sequences assigned to the (Firmicutes) $\mathrm{M} 2 \backslash \mathrm{M} 1$ group, the $\mathrm{RC}$ method classified five sequences in the Bacteroidetes phylum ( $\mathrm{Bc}>90 \%$ ) and not in the Firmicutes phylum. Moreover, of the 88 sequences assigned to the (Firmicutes) M1 $\backslash \mathrm{M} 2$ group, only four were classified as Firmicutes ( $\mathrm{BC}>90 \%$ ) using RC, suggesting the possibility of 84 false-positives as a consequence of DNA barcode application.

Thus, regardless of the method used, these results demonstrate the difficulty inherent to establishing the correct phylum classification for a small number of complete $16 \mathrm{~S}$ rRNA sequences.

\section{DNA barcodes applied to short I6S rRNA pyrosequencing reads}

Two pyrosequencing libraries generated in our laboratory for studies related to bacterial diversity in mixed microbial samples were analyzed. The first library was obtained from a brain abscess of a patient at a Marseille hospital and contained 2,612 reads with an average length of 95.78 base pairs. In parallel, results using classical Sanger sequencing of a $16 \mathrm{~S}$ rRNA clone library from the same sample were used as a source of comparison. We analyzed the $\mathrm{F} / \mathrm{B}$ ratios using two different culture-independent
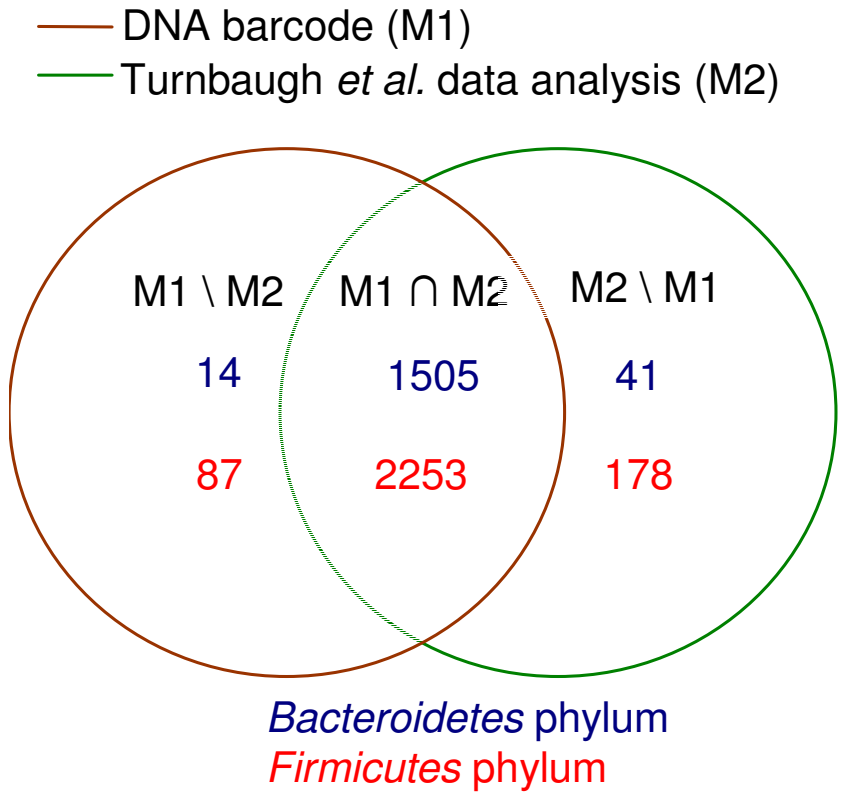

Figure 3

Discrepancies in sequence repartition of bacterial communities. $\mathrm{MI} \cap \mathrm{M} 2$ : In $\mathrm{MI}$ and $\mathrm{M} 2$; MIIM2: in $\mathrm{MI}$ out of $\mathrm{M} 2 ; \mathrm{M} 2 \mathrm{MI}$ : in $\mathrm{M} 2$ out of $\mathrm{MI}$. The blue and red figures are the number of sequences, respectively classified in Bacteroidetes and Firmicutes phyla by $\mathrm{MI}$ or $\mathrm{M} 2$, or by both of them.

methods: 16S rRNA pyrosequencing and 16S rRNA clonal Sanger sequencing. The results of the $16 \mathrm{~S}$ rRNA clonal Sanger sequencing method indicated that 50 of the clone sequences belonged to the Bacteroidetes phylum, while 49 belonged to the Firmicutes phylum, resulting in an F/B ratio of 0.98 (Table 4). Application of the DNA barcode method indicated that 41 of the pyrosequencing reads belonged to the Bacteroidetes phylum, while 39 belonged to the Firmicutes phylum, resulting in an $\mathrm{F} / \mathrm{B}$ ratio of 0.95 . We also analyzed the 2,612 reads using the sequence classification tool RDP-II classifier. The F/B ratio obtained using this tool was 1.02 (Table 4); however, $1.03 \%$ of all pyrosequencing reads obtained were too short in length to be classified by the RDP-II tool. In addition and contrary to our DNA barcode classification, the RDP-II classifier limits the number of sequences that can be submitted $(30,000$ per run). Finally, compared to the F/B ratio obtained with the RDP-II classifier tool (1.02), the ratio obtained with our DNA barcode method (0.95) was closer to that obtained by clonal Sanger sequencing (0.98).

The pyrosequencing error rate in the Bacteroidetes barcode sequence, which contains 4 homopolymers (GGGTTTAAAGGG), was estimated to $8.89 \%$ ( 4 errors/45) for this dataset. In all cases, the sequencing errors in the Bacteroidetes barcode sequence were due to a one base insertion 
Table 4: Determination of F/B ratios using pyrosequencing and clonal sequencing

\begin{tabular}{|c|c|c|c|c|c|}
\hline Sample & Sequencing method & Estimation method & $\boldsymbol{F}$ & B & ratio $\mathrm{F} / \mathrm{B}$ \\
\hline \multirow[t]{3}{*}{ Brain abscess pus } & I6S rRNA P & Barcode hits & 39 & 41 & 0.95 \\
\hline & I6S rRNA P & RDP-II classifier & 521 & 509 & 1.02 \\
\hline & I6S rRNA CS & Number of clone sequences & 49 & 50 & 0.98 \\
\hline \multirow[t]{2}{*}{ Cystic fibrosis sputum } & I6S rRNA P & Barcode hits & 34 & 2 & 17 \\
\hline & I6S rRNA CS & Number of clone sequences & 16 & 1 & 16 \\
\hline \multirow[t]{2}{*}{ Caecal content } & MP & Barcode hits & 79 & 33 & 2.39 \\
\hline & & $\%$ of total $16 \mathrm{~S}$ rRNA sequences & 60.70 & 28.41 & 2.14 \\
\hline
\end{tabular}

P, CS and MP mean Pyrosequencing, Clonal Sanger Sequencing and Metagenome Pyrosequencing, respectively. F and B mean Firmicutes phylum and Bacteroidetes phylum, respectively. The numbers of clone sequences assigned to the Firmicutes or Bacteroidetes phylum was performed using BLAST algorithm and Genebank database. For the metagenome pyrosequencing, the I6S rRNA fraction was found with BLAST algorithm and RDP-II database. RDP-II classifier was used as a control with a bootstrap confidence $>80 \%$

(A) in the homopolymer A. One of these cases also showed a one base insertion ( $\mathrm{T})$ in the homopolymer $\mathrm{T}$ (Table 5).

A second pyrosequencing library from the sputum of a cystic fibrosis (CF) patient from a Marseille hospital was also analyzed. This library contained 4,499 reads with an average length of 93.05 base pairs. A 16S rRNA clonal Sanger sequencing approach was also applied to the same CF sputum. The results of the two culture-independent methods were similar, as indicated in Table 4. Results from the clonal Sanger sequencing analysis indicated that 16 clone sequences belonged to the Firmicutes phylum, while only one sequence was assigned to the Bacteroidetes phylum $(\mathrm{F} / \mathrm{B}=16)$. Analysis of the $16 \mathrm{~S}$ rRNA pyrosequencing data indicated that the Firmicutes DNA barcode matched 34 sequence reads, while the Bacteroidetes DNA barcode matched two sequence reads $(\mathrm{F} / \mathrm{B}=17)$.

\section{DNA barcodes applied to metagenome pyrosequencing} From the metagenomic study performed by Turnbaugh $e t$ al.[2], 1,046,611 and 677,384 pyrosequencing reads were collected from Lean 1 and Obese1 mice, respectively, using the GS 20 pyrosequencer. The authors analyzed the merged data $(1,723,995$ reads) by BLASTing against the $16 S$ rRNA RDP-II database. Using this method, the authors determined the total number of sequence reads classified as $16 \mathrm{~S}$ rRNA genes and the number of sequence reads belonging to Bacteroidetes and Firmicutes phyla. This analysis led to the determination of an F/B ratio of 2.14. Using our DNA barcode method with this dataset assigned 79 reads to the Firmicutes phylum and 33 reads to the Bacteroidetes phylum, resulting in an $\mathrm{F} / \mathrm{B}$ ratio of 2.39 (Table 4).

\section{Discussion}

Owing to the rapid accumulation of data coupled with advances in sequencing technology - including the 454 Life Sciences GS FLX System sequencer [6], which generates more than 100 million bases per run - the development of methods capable of rapidly processing these data has become essential. Thus, the aim of the present investigation was to provide a straightforward, accurate, inexpensive and rapid tool to estimate the relative abundance of bacterial communities and the resulting $\mathrm{F} / \mathrm{B}$ ratios from thousands of $16 \mathrm{~S}$ rRNA short sequencing reads, without the need for any assembly procedure, multiple sequence alignment, BLAST analysis, or phylogeny reconstruction.

Table 5: Pyrosequencing errors

\begin{tabular}{|c|c|}
\hline Read label & Pyrosequencing errors in Bacteroide \\
\hline 002964_0126_2423 & GGGTTTAAAAGGG \\
\hline 000955_0116_2845 & GGGTTTAAAAGGG \\
\hline 003I77_0II2_248I & GGGTTTAAAAGGG \\
\hline $001840 \_0197 \_1892$ & GGGTTTTAAAAGGG \\
\hline
\end{tabular}

Sequencing errors found in the Bacteroidetes barcode 'GGGTTTAAAGG' using the brain abscess pyrosequencing dataset. The sequencing errors were identified as an $A(4 / 4)$ and a $T(1 / 4)$ base insertion. 
The DNA barcode obtained for the Bacteroidetes phylum is sensitive and specific. In the literature, Dick and Field reported a $16 \mathrm{~S}$ rRNA Bacteroidetes barcode that can be used as a probe to estimate the occurrence of fecal Bacteroidetes [19]. As observed with our DNA barcode using the RDP-II database, assessment of the sensitivity and specificity of the Bacteroidetes DNA barcode reported by Dick and Field produced a sensitivity score of $88.00 \%$ (compared to 96.52\% for our Bacteroidetes barcode) and a specificity score of a $99.25 \%$ (compared to $99.98 \%$ for our Bacteroidetes barcode). Thus, our Bacteroidetes barcode possesses both greater sensitivity and greater specificity. Although the Fusobacteria and Cyanobacteria phyla are responsible for a slight decrease in the specificity of our Firmicutes barcode, the relative proportion of both of these phyla is marginal (less than $0.15 \%$ of all $16 \mathrm{~S}$ rRNA sequences in each 16S rRNA survey [1-4]).

Compared to the nearly completed 16S rRNA sequences assembled from four clone library surveys of gut microbiota, the results obtained using our 16S rRNA barcode application are in agreement $(\mathrm{P}>0.05)$. Importantly, there are some discrepancies that highlight several limitations of the bioinformatics and biological methodologies. First, it is possible that some sequences belonging to the Firmicutes or Bacteroidetes phyla may not be identified using our method since the sensitivity of our DNA barcode is less than $100 \%$. Moreover, due to limited specificity (less than $100 \%$ for our DNA barcode), some falsepositives may be introduced into the results. Another discrepancy is caused by the requirement for polymerase chain reaction and its capacity to generate sequencing errors. A single base sequencing error (substitution or deletion) located in the region of a conserved nucleotide comprising the DNA barcode may result in a false-positive or false-negative result. The quality of the sequencing analysis and the sequence assembly process are important to the quality of the results obtained using the DNA barcode method.

The results provided by the RDP-II "classifier" taxonomic assignment method suggest that there are few errors in the taxonomic classification of the 16S rRNA sequences from the clone libraries and emphasize the need to benchmark the tools used in such analyses. To estimate the abundance of phyla within a bacterial community using the phylogenetic reconstruction ARB tool, 16S rRNA surveys of the intestinal microbial flora first target the computation of a multiple sequence alignment (MSA) with the NAST multialigner [20] or the autoaligner of the ARB software. It is obvious that the quality of the phylogenetic reconstruction is directly related to the accuracy of the sequence alignment analysis. In many cases, an alignment is considered biologically satisfactory when it accurately reflects the structural relationship between the given sequences. As a consequence, MSA algorithms are typically benchmarked with a collection of structure-based sequence alignments $[21,22]$, which are considered to be gold standards [23]. In contrast, the processing methods involved in 16S rRNA studies suffer from a lack of benchmarking tests or gold standard references [24]. Thus, the recent MSA programs $[18,20]$ capable of aligning large quantities of $16 \mathrm{~S}$ rRNA sequences must be evaluated for their alignment accuracy, as currently performed for Muscle, T-COFFEE, MAFFT, Probcons, and the Clustalw MSA programs for protein sequences $[25,26]$. Recently, Carroll et al. [27] proposed the first DNA database of 3,545 DNA reference alignments. Finally, because the accuracy of phylogeny reconstruction depends on the number of informative sites, the short pyrosequencing reads collected by the GS 20 are theoretically not suitable for inferring phylogenic reconstruction. In a study performed by Zongzhi et al. [14], however, alignment of short pyrosequencing reads by NAST and insertion of these sequences into a preestablished phylogenic tree of full-length 16S rRNA gene sequences using ARB provided satisfactory results. While laborious data analyses involving sequencing of the $16 \mathrm{~S}$ rRNA clone library enable the characterization of bacterial taxonomy at the species level, they do not represent an effective, low-cost strategy for clinical diagnosis or for monitoring $\mathrm{F} / \mathrm{B}$ ratio variations in a large spectrum of obese humans.

In contrast, $16 \mathrm{~S}$ rRNA or metagenome pyrosequencing offers an inexpensive and rapid strategy that can exploit the use of a DNA barcode representative of a bacterial phylum to process thousands of short sequence reads. The short read lengths of pyrosequencing are sufficient to successfully estimate $\mathrm{F} / \mathrm{B}$ ratios using the DNA barcode method, but because our Bacteroidetes barcode contains homopolymers and because errors in pyrosequencing reads (indels and ambiguous bases) occur most often in homopolymeric regions [28], the proportion of Bacteroidetes can be underestimated (Table 5). Finally, we assume a similar pyrosequencing error rate in the Bacteroidetes and in the Firmicutes barcodes since the F/B ratios obtained with the pyrosequencing and the clonal Sanger sequencing data were closed (Table 4).

A short pattern search against a significantly large sequence database (e.g., metagenome data) is less timeconsuming with respect to $\mathrm{CPU}$ time and much faster than performing the BLAST search algorithm against a $16 \mathrm{~S}$ rRNA database. Moreover, results from the BLAST analysis require that the identified species be grouped by phylum using a tool such as the ARB tool.

The evolution of pyrosequencing strategies has focused on targeting of specific regions [14] and maximizing multiplexing capabilities (massive parallel or barcoding pyrose- 
quencing), allowing independent samples to be pooled together and sequenced in a single run due to a short tag carried at the 5 'end of the primer $[5,15]$. Since the DNA barcodes for Firmicutes and Bacteroidetes are separated by approximately $600 \mathrm{bp}$, they cannot be sequenced in the same read using pyrosequencing technology. An alternative method using distinct DNA amplification (with two primer sets) of the $16 \mathrm{~S}$ rRNA region of Bacteroidetes and Firmicutes barcodes would not be an effective strategy due to differences in amplification efficiency [29]. In the near future, however, rapid technical advances ( 400-500 bases for the next-generation GS FLX titanium instrument) will likely increase the read length and overcome this drawback, which currently prevents massive parallel and specific region pyrosequencing strategies.

Finally, these DNA barcodes should permit the development of real-time PCR assays using the barcode as a probe. This will be an elegant, low cost, and effective application for day-to-day use in clinical settings.

\section{Conclusion}

Based on a DNA barcode for 16S rRNA gene sequences, we have proposed a useful and practical, yet low cost strategy to effectively evaluate obesity in humans. This is accomplished using a method that rapidly determines the $\mathrm{F} / \mathrm{B}$ ratio present in a patient. Our DNA barcodes target the two major phyla of the gastrointestinal bacterial community, which show changes in their abundance in obese humans. Additionally, these DNA barcodes are capable of rapidly processing thousands of short sequencing reads. The F/B ratios that result from 16S rRNA clonal Sanger sequencing, 16S rRNA pyrosequencing, and metagenome pyrosequencing can be accurately estimated using our DNA barcodes.

The short length of the reads that result from highthroughput pyrosequencing coupled with the high degree of nucleotide conservation among the 16S rRNA genes prevents sequence assembly. Consequently, short DNA fragments exploited as DNA tags or barcodes that enable the characterization of taxonomy at the phylum, genus, or species level represent tools that are adapted to assist in clinical diagnosis and monitoring relevant changes in the relative abundance of bacterial communities in microbial ecosystems.

\section{Methods}

\section{I6S rRNA RDP-II and Greengenes databases}

The 16S rRNA sequences of the bacterial phyla were downloaded from the Ribosomal Database Project-II site [30]. Both "isolates" and "uncultured" sequences greater than 1,200 bp were selected. Only those sequences defined as "Good Quality" were retrieved. From the
Greengenes database [31], the sequences of the bacterial phyla were exported using the NCBI taxonomy.

\section{Full I6S rRNA sequences retrieved from clonal Sanger sequencing datasets}

The 16S rRNA datasets (sequences, alignment, and phylogenetic tree) obtained from the Ley et al. 16S rRNA surveys $[3,4]$ were retrieved from an ARB file located at [32] and [33]. For each ARB file, the sequences assigned to Firmicutes and Bacteroidetes were extracted and imported in Fasta file format. The relative abundance of the major bacterial communities of all complete 16S rRNA gene studies was calculated from the Fasta format files. It was necessary to install the ARB software package [34] to access these data. The $16 \mathrm{~S}$ rRNA dataset (sequences, alignment, and phylogenetic tree) obtained from the Eckburg et al. study [1] were downloaded from [35]. Complementary results were obtained upon request. The last 16S rRNA dataset (sequences, alignment, and phylogenetic tree) acquired from the Turnbaugh et al. study [2] was selected from [36].

\section{DNA barcode identification}

The general procedure used to define the final DNA barcode is described in the flow chart in Figure 4. Extraction of the $\mathrm{N}$ most representative sequences was performed with the Seq_reformat program, a part of the T-COFFEE package [37]. This extraction discarded sequence redundancy and reduced the time calculation for pattern search. The MEME search motif program [38] was installed locally and run using default options. The patterns were tested against the 16S rRNA sequences of the databases using Dreg, an EMBOSS package program. For Firmicutes, the final barcode was refined manually via multiple sequence alignments of the phylum. Muscle version 3.56 was performed for the multiple sequence alignment (MSA) and Seaview [39] executable for the MSA edition.

\section{Evaluation of DNA barcodes with reduced I 6S rRNA data}

The Firmicutes signature was not applied to the dataset obtained from the Ley et al. study [3] because more than 1,500 sequences of the 5,088 had a length inferior to 1,200 bp. Similarly, the dataset obtained from the Ley et al. study [4] was reduced from 18,348 to 16,615 sequences because several sequences were too short to potentially possess the Firmicutes barcode.

\section{Sensitivity and specificity of DNA barcodes}

The sensitivity of the DNA barcode was defined as the fraction of $16 \mathrm{~S}$ rRNA sequences of the phylum that possessed the barcode. The specificity of the DNA barcode was defined as the fraction of sequences of a phylum that did not possess the barcode. 


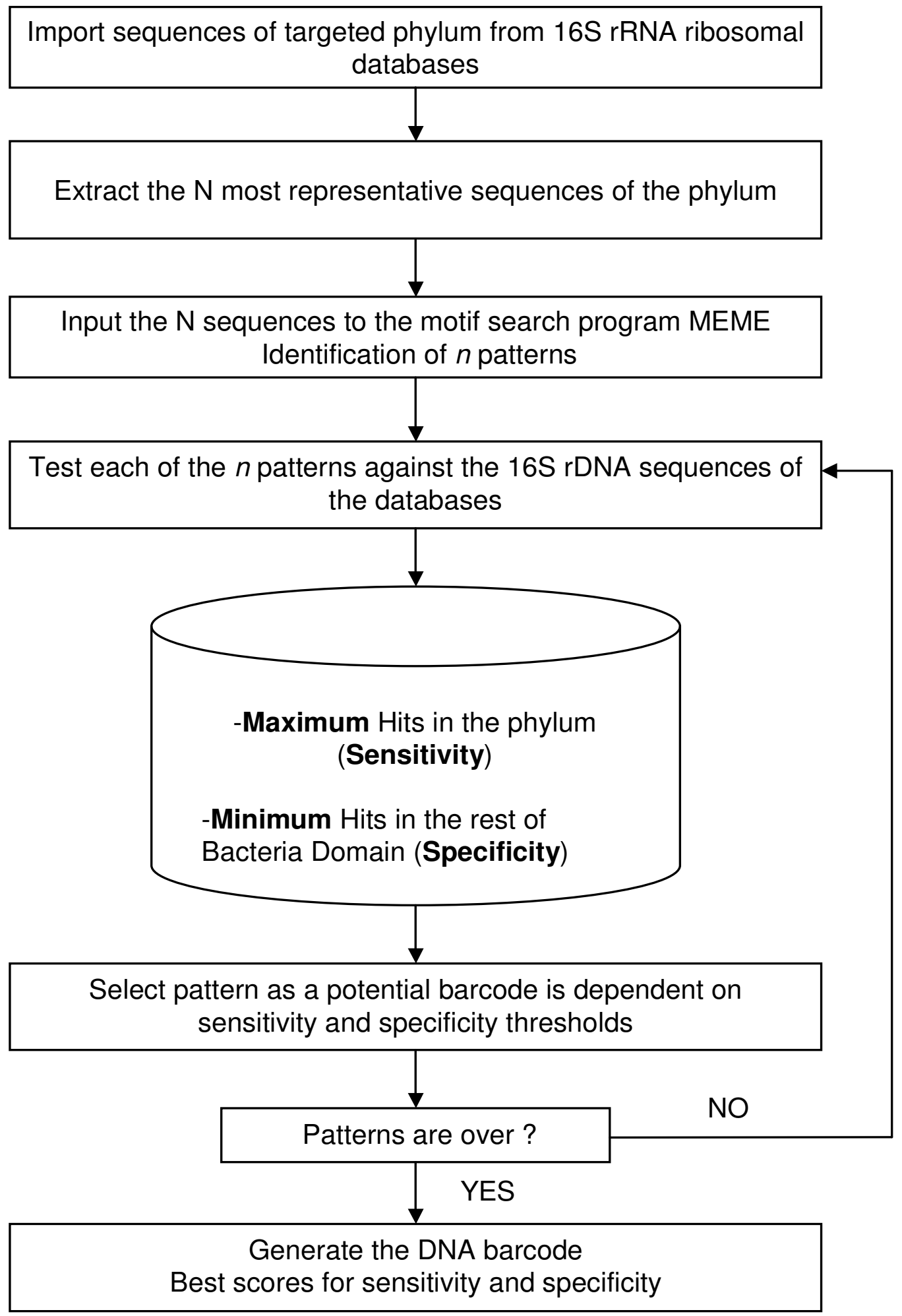

Figure 4

Flow chart summarizing the procedure for identifying the DNA barcodes. 


\section{Taxonomic assignment}

We used a Naïve Bayesian rRNA classifier [40] to compare discrepancies between the results of our DNA barcode method and those of $16 \mathrm{~S}$ rRNA surveys [1-4].

\section{Discrepancies}

M1 represents the DNA barcode method used herein, and M2 is the method applied in the 16S rRNA studies [1-4]. M1 n M2 was the number of sequences identically classified by both methods. The $n$ (Firmicutes) $\mathrm{M} 1 \backslash \mathrm{M} 2$ indicates $n$ sequences were assigned to Firmicutes phylum by $\mathrm{M} 1$, but not by M2. The $n$ (Bacteroidetes) M2 $\backslash \mathrm{M} 1$ indicates $n$ sequences were assigned to the Bacteroidetes phylum by M2, but not by M1.

\section{Clonal Sanger sequencing libraries}

The DNA extraction, genomic amplification, cloning procedures, and sequencing are described in Bittar et al. [41]. From the brain abscess cerebral sample, a library of 100 sequencing clones was analyzed. From the cystic fibrosis sputum, a library of 36 sequencing clones was analyzed.

\section{Sequencing errors in the Bacteroidetes barcode}

Identification of sequencing errors in the Bacteroidetes barcode was performed by BLAST similarity search against the pyrosequencing dataset of the brain abscess sample using an extended barcode consensus sequence (CCGGANTTATTGGGTTTAAAGGGNGCG) from all the Bacteroidetes sequences identified by clonal Sanger sequencing. Reads classified as member of the Bacteroidetes phylum by RDP-II classifier (BP > 95) and with sequencing errors in the Bacteroidetes barcode were labelled 002964_0126_24 23,000955_0116_2845,003177_0112_2481 and 00184 0_0197_1892.

\section{Pyrosequencing libraries}

The 16S pyrosequencing sequences from cystic fibrosis sputum were deposited in the NCBI Short Read Archive under accession number SRS001099 and taxid 433733. The 16S pyrosequencing sequences from the pus of a brain abscess were deposited in the NCBI Short Read Archive under accession number SRS001098 and taxid 539654. Following the conditions detailed in Margulies et al. [6], PCR products (the amplicon size is about $1460 \mathrm{bp}$ ) were sequenced with the GS 20 platform (454 Life Science-Roche) using a titration $40 \times 75$ Picotitreplate $^{\mathrm{TM}}$ (PTP) with eight regions. Four conditions were tested and duplicated as described by the Roche procedure, and bioinformatics analysis was performed for the dataset from the region that was closest to the optimal condition (accession number SRS001099 and SRS001098) of one DNA copy per bead.

\section{Competing interests}

The authors declare that they have no competing interests.

\section{Authors' contributions}

FA designed and performed all data analyses and wrote the first draft of the manuscript. DR conceived of the study and helped draft the manuscript. Both authors read and approved the final version of the manuscript.

\section{Funding}

This work was funded by the Network of Excellence European Pathogenomics.

\section{Acknowledgements}

We thank Pierre-Edouard Fournier for useful discussions and suggestions during the course of this work. We thank Christelle Desnues for comments regarding and corrections of the manuscript.

\section{References}

I. Eckburg PB, Bik EM, Bernstein CN, Purdom E, Dethlefsen L, Sargent $M$, et al.: Diversity of the human intestinal microbial flora. Science 2005, 308:1635-1638.

2. Turnbaugh PJ, Ley RE, Mahowald MA, Magrini V, Mardis ER, Gordon II: An obesity-associated gut microbiome with increased capacity for energy harvest. Nature 2006, 444:|027-I03I.

3. Ley RE, Backhed F, Turnbaugh P, Lozupone CA, Knight RD, Gordon II: Obesity alters gut microbial ecology. Proc Natl Acad Sci USA 2005, 102: I 1070-I 1075.

4. Ley RE, Turnbaugh PJ, Klein S, Gordon Jl: Microbial ecology: human gut microbes associated with obesity. Nature 2006, 444: $1022-1023$

5. Andersson AF, Lindberg M, Jakobsson H, Backhed F, Nyren P, Engstrand L: Comparative analysis of human gut microbiota by barcoded pyrosequencing. PLoS ONE 2008, 3:e2836.

6. Margulies M, Egholm M, Altman WE, Attiya S, Bader JS, Bemben LA, et al.: Genome sequencing in microfabricated high-density picolitre reactors. Nature 2005, 437:376-380.

7. Huber JA, Mark Welch DB, Morrison HG, Huse SM, Neal PR, Butterfield DA, et al:: Microbial population structures in the deep marine biosphere. Science 2007, 3 I 8:97-100.

8. Sogin ML, Morrison HG, Huber JA, Mark WD, Huse SM, Neal PR, et al.: Microbial diversity in the deep sea and the underexplored "rare biosphere". Proc Natl Acad Sci USA 2006, I 03: I 2 I I5-I 2 I 20.

9. Roesch LF, Fulthorpe RR, Riva A, Casella G, Hadwin AK, Kent AD, et al.: Pyrosequencing enumerates and contrasts soil microbial diversity. ISME J 2007, I:283-290.

10. Hugenholtz P, Tyson GW: Microbiology: metagenomics. Nature 2008, 455:48I-483.

II. Wang Q, Garrity GM, Tiedje JM, Cole JR: Naive Bayesian classifier for rapid assignment of rRNA sequences into the new bacterial taxonomy. Appl Environ Microbiol 2007, 73:526I-5267.

12. Krause L, Diaz NN, Goesmann A, Kelley S, Nattkemper TW, Rohwer F, et al: Phylogenetic classification of short environmental DNA fragments. Nucleic Acids Res 2008, 36:2230-2239.

13. Sundquist A, Bigdeli S, Jalili R, Druzin ML, Waller S, Pullen KM, et al.: Bacterial flora-typing with targeted, chip-based Pyrosequencing. BMC Microbiol 2007, 7:108.

14. Liu Z, Lozupone C, Hamady M, Bushman FD, Knight R: Short pyrosequencing reads suffice for accurate microbial community analysis. Nucleic Acids Res 2007, 35:e I 20

15. McKenna P, Hoffmann C, Minkah N, Aye PP, Lackner A, Liu Z, et al.: The macaque gut microbiome in health, lentiviral infection, and chronic enterocolitis. PLoS Pathog 2008, 4:e20.

16. Cole JR, Chai B, Farris RJ, Wang Q, Kulam-Syed-Mohideen AS, McGarrell DM, et al.: The ribosomal database project (RDP-II): introducing myRDP space and quality controlled public data. Nucleic Acids Res 2007, 35:DI69-DI72.

17. Desantis TZ, Hugenholtz P, Larsen N, Rojas M, Brodie EL, Keller K, et al:: Greengenes, a chimera-checked I6S rRNA gene database and workbench compatible with ARB. Appl Environ Microbiol 2006, 72:5069-5072.

18. Ludwig W, Strunk O, Westram R, Richter L, Meier H, Yadhukumar, et al.: ARB: a software environment for sequence data. Nucleic Acids Res 2004, 32:1363-1371. 
19. Dick LK, Field KG: Rapid estimation of numbers of fecal Bacteroidetes by use of a quantitative PCR assay for I6S rRNA genes. Appl Environ Microbiol 2004, 70:5695-5697.

20. DeSantis TZ Jr, Hugenholtz P, Keller K, Brodie EL, Larsen N, Piceno YM, et al.: NAST: a multiple sequence alignment server for comparative analysis of 16S rRNA genes. Nucleic Acids Res 2006, 34:W394-W399.

21. Thompson JD, Plewniak F, Poch O: BAliBASE: a benchmark alignment database for the evaluation of multiple alignment programs. Bioinformatics 1999, 15:87-88.

22. Van WI, Lasters I, Wyns L: SABmark - a benchmark for sequence alignment that covers the entire known fold space. Bioinformatics 2005, 21: 1267-1 268.

23. Armougom F, Moretti S, Keduas V, Notredame C: The iRMSD: a local measure of sequence alignment accuracy using structural information. Bioinformatics 2006, 22:e35-e39.

24. Mavromatis K, Ivanova N, Barry K, Shapiro H, Goltsman E, McHardy AC, et al.: Use of simulated data sets to evaluate the fidelity of metagenomic processing methods. Nat Methods 2007, 4:495-500.

25. Katoh K, Kuma K, Toh H, Miyata T: MAFFT version 5: improvement in accuracy of multiple sequence alignment. Nucleic Acids Res 2005, 33:5। I-518.

26. Edgar RC: MUSCLE: multiple sequence alignment with high accuracy and high throughput. Nucleic Acids Res 2004, 32: $1792-1797$

27. Carroll H, Beckstead W, O'Connor T, Ebbert M, Clement M, Snell Q, et al.: DNA reference alignment benchmarks based on tertiary structure of encoded proteins. Bioinformatics 2007 23:2648-2649.

28. Eriksson N, Pachter L, Mitsuya Y, Rhee SY, Wang C, Gharizadeh B, et al.: Viral population estimation using pyrosequencing. PLoS Comput Biol 2008, 4:el000074.

29. Schmalenberger A, Schwieger F, Tebbe CC: Effect of primers hybridizing to different evolutionarily conserved regions of the small-subunit rRNA gene in PCR-based microbial community analyses and genetic profiling. Appl Environ Microbiol 2001, 67:3557-3563.

30. The Ribosomal Database Project-II site [http:// rdp.cme.msu.edu/]

31. The Greengenes Database [http://greengenes.lbl.gov/]

32. Ley et al. I6S rRNA mice sequences [http://gordon lab.wustl.edu/mice]

33. Ley et al. I6S rRNA human sequences [http://gordon lab.wustl.edu/microbial ecology human obesity]

34. ARB software package [http://www.arb-home.de/]

35. Eckburg et al. I6S rRNA sequences [http://arb-db-cen tral.swiki.net/l]

36. Turnbaugh et al. I6S rRNA sequences [http://gordon lab.wustl.edu/supplemental/Turnbaugh/obob/]

37. Notredame C, Higgins DG, Heringa J: T-Coffee: A novel method for fast and accurate multiple sequence alignment. J Mol Biol 2000, 302:205-217.

38. Bailey TL, Williams N, Misleh C, Li WW: MEME: discovering and analyzing DNA and protein sequence motifs. Nucleic Acids Res 2006, 34:W369-W373.

39. Galtier N, Gouy M, Gautier C: SEAVIEW and PHYLO_WIN: two graphic tools for sequence alignment and molecular phylogeny. Comput Appl Biosci 1996, 12:543-548.

40. Bayesian rRNA classifier [http://rdp.cme.msu.edu/classifier/clas sifier.jsp]

4I. Bittar F, Richet H, Dubus JC, Reynaud-Gaubert M, Stremler N, Sarles J, et al.: Molecular detection of multiple emerging pathogens in sputa from cystic fibrosis patients. PLOS ONE 2008, 3:e2908.
Publish with Bio Med Central and every scientist can read your work free of charge

"BioMed Central will be the most significant development for disseminating the results of biomedical research in our lifetime. "

Sir Paul Nurse, Cancer Research UK

Your research papers will be:

- available free of charge to the entire biomedical community

- peer reviewed and published immediately upon acceptance

- cited in PubMed and archived on PubMed Central

- yours - you keep the copyright
BioMedcentral 